\title{
Bisectors of Linearly Separable Sets
}

\author{
Lee R. Nackman and Vijay Srinivasan \\ Manufacturing Research Department, IBM Research Division, \\ Thomas J. Watson Research Center, P.O. Box 218, \\ Yorktown Heights, NY 10598, USA
}

\begin{abstract}
A bisector of two sets is the set of points equidistant from them. Bisectors arise naturally in several areas of computational geometry. We show that bisectors of weakly linearly separable sets in $E^{d}$ have many properties of interest. Among these, the bisector of a restricted class of linearly separated sets is a homeomorphic image of the linear separator. We also give necessary and sufficient conditions for the existence of a particular continuous map from (a portion of any linear separator to the bisector.
\end{abstract}

\section{Introduction}

Bisectors, which are defined as the set of points equidistant from two given sets, arise naturally in computing the symmetric axis transform [1] and in computing Voronoi diagrams [5-9]. For example, a common means of computing the Voronoi diagram is the following recursive approach: compute separately the Voronoi diagrams of two subproblems and then merge them together; during this merge, the bisector of the two subproblems is used to "trim" the Voronoi diagrams of the subproblems. Certain properties of the bisector are the key to an efficient merge. For example, one algorithm for computing the Voronoi diagram of point sites in $E^{2}$ using a divide-and-conquer strategy [8] partitions the points into two almost equal-sized sets separated by a line. The bisector between these two sets is connected and is a single-valued map of the dividing line. In the case of computing the Voronoi diagram of multiply connected polygonal domains [9], certain bisectors are simple, closed curves. In each of these two examples, properties of the bisector allow a linear-time merge. Moreover, knowing the topology of the bisector helps in choosing an appropriate data structure for the bisector. For example, if the bisector of two sets in $E^{3}$ is known to be a 2-manifold, then the QuadEdge data structure [2] can be used to represent and manipulate it.

In this paper we show that bisectors of linearly separable sets have many 
properties of interest. The results presented here are for general sets in $E^{d}$. There are several reasons for this. First, many of the results and proofs are simpler when the details of a particular class of sets do not intrude. More importantly, we want to broaden the study of bisectors beyond their use in algorithms for computing the Voronoi diagram of point sites in $E^{2}$ because we believe that proximity properties of more general geometric elements in higher dimensions have important applications.

\section{Linearly Separable Sets}

We denote the closure of a set $S$ by cl $S$, the interior by int $S$, the boundary by $\partial S$, and the closure of the convex hull of $S$ by $\mathrm{CH}(S)$. Boldface lowercase characters denote points in $E^{d}$ and $p_{i}$ denotes the $i$ th coordinate of a point $\mathbf{p}$.

The (Euclidean) distance between two points $\mathbf{p}$ and $\mathbf{q}$ is denoted by $d(\mathbf{p}, \mathbf{q})$. The distance between a point $\mathbf{p}$ and a nonempty set $S$ is $d(\mathbf{p}, S)=\operatorname{glb}\{d(\mathbf{p}, \mathbf{q}): q \in S\}$. The nearness of $S_{1}$ and $S_{2}$ is $n\left(S_{1}, S_{2}\right)=\operatorname{glb}\left\{d(\mathbf{p}, \mathbf{q}): \mathbf{p} \in S_{1}, \mathbf{q} \in S_{2}\right\}$. Let $\operatorname{Sl}\left(\pi_{1}, \pi_{2}\right)$ denote the open slab between the two distinct parallel hyperplanes $\pi_{1}$ and $\pi_{2} . S_{1}$ and $S_{2}$ are separated by a slab $\operatorname{Sl}\left(\pi_{1}, \pi_{2}\right)$ if $S_{1}$ and $S_{2}$ lie in different components of $E^{d}-\operatorname{Sl}\left(\pi_{1}, \pi_{2}\right) . S_{1}$ and $S_{2}$ are strongly linearly separable if there exists an open slab that separates cl $S_{1}$ and $\mathrm{cl} S_{2}$. A hyperplane contained in such an open slab is called a strong linear separator. $S_{1}$ and $S_{2}$ are linearly separable if there exists a hyperplane $\pi$, called a linear separator, such that $\mathrm{cl} S_{1}$ and $\mathrm{cl} S_{2}$ are in different components of $E^{d}-\pi$. Similarly, $S_{1}$ and $S_{2}$ are weakly linearly separable if there exists a
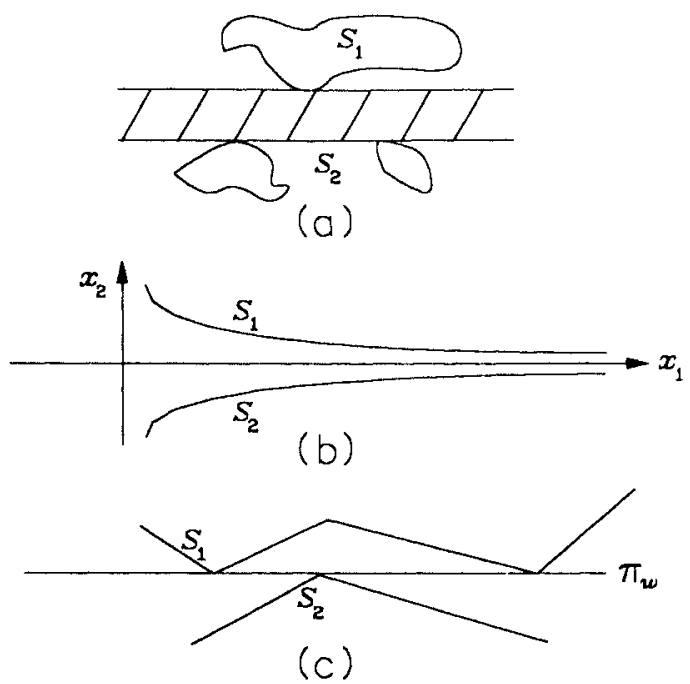

Fig. 1. Three types of linear separability. (a) $S_{1}$ and $S_{2}$ are strongly linearly separable since they are separated by the cross-hatched slab. (b) $S_{1}: x_{2}=x_{1}^{-1}, S_{2}: x_{2}=-x_{1}^{-1}, x_{1}>0$, are linearly separable (but not strongly linearly separable) and $x_{2}=0$ is the linear separator. (c) $S_{1}$ and $S_{2}$ are weakly linearly separable (but not linearly separable) and $\pi_{\mathrm{w}}$ is the weak linear separator. 
hyperplane $\pi_{\mathrm{w}}$ called a weak linear separator, such that $\mathrm{cl} S_{1}$ and $\mathrm{cl} S_{2}$ lie in the closures of different components of $E^{d}-\pi$. Figure 1 shows some examples in $E^{2}$ of separable sets and separators.

Note that strongly linearly separable sets arc also linearly separable and linearly separable sets are also weakly linearly separable. Likewise, a strong linear separator is also a linear separator and a linear separator is also a weak linear separator. Most results presented in this paper are formulated in terms of weakly linearly separable sets and weak linear separators.

A practically useful characterization of strong linear separability is given by:

Theorem 1. If at least one of $S_{1}$ and $S_{2}$ is bounded, then $S_{1}$ and $S_{2}$ are strongly linearly separable if and only if $\mathrm{CH}\left(S_{1}\right) \cap \mathrm{CH}\left(S_{2}\right)=\varnothing$.

Proof. Since at least one of $S_{1}$ and $S_{2}$ is bounded, $\mathrm{CH}\left(S_{1}\right) \cap \mathrm{CH}\left(S_{2}\right)=\varnothing$ implies that $n\left(\mathrm{CH}\left(S_{1}\right), \mathrm{CH}\left(S_{2}\right)\right)>0$ (Corollary 7.2, Section 1.8, of [4]). Since the nearness is positive, there exists a slab that separates $\mathrm{CH}\left(S_{1}\right)$ and $\mathrm{CH}\left(S_{2}\right)$ (Theorem 4, Section 6.2, of [4]). $S_{1}$ and $S_{2}$ are therefore strongly linearly separable. The converse is trivial.

\section{Bisectors}

In this section we investigate properties of the bisector of two weakly linearly separable sets $S_{1}$ and $S_{2}$. These are of use in Section 4 in studying the topology of the bisector. Hereafter, we assume that $S_{1}$ and $S_{2}$ are nonempty sets in $E^{d}$ such that cl $S_{1} \cap \mathrm{cl} S_{2}=\varnothing$.

The bisector $B\left(S_{1}, S_{2}\right)$ of two sets $S_{1}$ and $S_{2}$ is the set of points equidistant from $S_{1}$ and $S_{2}$, i.e., $B\left(S_{1}, S_{2}\right)=\left\{\mathbf{p} \in E^{d}: d\left(\mathbf{p}, S_{1}\right)=d\left(\mathbf{p}, S_{2}\right)\right\}$. Let $\mathbf{q}$ be a point on $B\left(S_{1}, S_{2}\right)$ and define the maximal ball $\mathscr{B}$ to be the open ball centered at $\mathbf{q}$ with radius $r=d\left(\mathbf{q}, S_{1}\right)=d\left(\mathbf{q}, S_{2}\right)$. Also define the maximal sphere $\mathscr{S}=\partial \mathscr{H}$. Observe that, by definition, $\mathscr{B}$ does not contain points of $S_{1}$ or $S_{2}$ and that $\mathscr{S}$ contains at least one point $\mathbf{p}_{1}$ from cl $S_{1}$ and at least one point $\mathbf{p}_{2}$ from $\mathrm{cl} S_{2}$. The points $\mathbf{p}_{1}$ and $\mathrm{p}_{2}$ are called touching points of $\mathscr{S}$ on $S_{1}$ and $S_{2}$, respectively, and $\mathscr{S}$ is said to touch $S_{1}$ and $S_{2}$. Notice that $\mathbf{p}_{1} \neq \mathbf{p}_{2}$ as cl $S_{1} \cap \mathrm{cl} S_{2}=\varnothing$. One maximal ball cannot contain another because the included maximal sphere would lack touching points.

Without loss of generality, we assume that a weak linear separator $\pi_{w}$ is the hyperplane $x_{d}=0$, that $S_{1}$ is contained in the closure of the open half-space $\pi_{w}^{1}: x_{d}>0$, and that $S_{2}$ is contained in the closure of the open half-space $\pi_{w}^{2}: x_{d}<0$. The mapping we consider takes a point on a weak linear separator vertically up or down to a point in the bisector. We first show in Section 3.1 that if a line perpendicular to $\pi_{\mathrm{w}}$ intersects $B\left(S_{1}, S_{2}\right)$, then the intersection is connected. Then, in Section 3.2 , we establish the necessary and sufficient conditions under which all lines perpendicular to $\pi_{\mathrm{w}}$ intersect $B\left(S_{1}, S_{2}\right)$. Finally, in Section 3.3 , we give the necessary and sufficient conditions for a specific line perpendicular to $\pi_{\mathrm{w}}$ to intersect $B\left(S_{1}, S_{2}\right)$. 


\subsection{Connectedness of Intersection with a Perpendicular}

Theorem 2. If a line l perpendicular to a weak linear separator $\pi_{\mathrm{w}}$ of $S_{1}$ and $S_{2}$ intersects $B\left(S_{1}, S_{2}\right)$, then it does so in a connected subset of l. Moreover, if a point $\mathbf{q}$ is in the relative interior of $\ln B\left(S_{1}, S_{2}\right)$, then all the touching points of the maximal sphere centered at $\mathbf{q}$ are in $\pi_{w}$.

Proof. To show that the intersection is connected, assume that there are two distinct points $\mathbf{q}_{a}$ and $\mathbf{q}_{b}$ in $\ln B\left(S_{1}, S_{2}\right)$. Let $\mathscr{B}_{a}, \mathscr{S}_{a}, \mathscr{B}_{b}$, and $\mathscr{S}_{b}$ be the corresponding maximal balls and spheres. Since $\mathbf{q}_{a}$ and $\mathbf{q}_{b}$ are distinct, $\mathscr{B}_{a} \neq \mathscr{B}_{b}$. Since one maximal ball cannot be contained in another, there are three remaining cases:

Case 1: $\mathscr{S}_{a}$ and $\mathscr{S}_{b}$ are disjoint. Since $\mathbf{q}_{a}$ and $\mathbf{q}_{b}$ are in $l$, there exists a hyperplane $\lambda$ perpendicular to $l$ that is a strong linear separator of $\mathscr{S}_{a}$ and $\mathscr{S}_{b}$. See Fig. 2(a). Since $\lambda$ is parallel to $\pi_{w}, \mathscr{S}_{a}$ cannot touch $S_{2}$ and/or $\mathscr{S}_{b}$ cannot touch $S_{1}$-a contradiction.

Case 2: $\mathscr{S}_{a}$ and $\mathscr{S}_{b}$ intersect at one point p. Let $\lambda$ be the hyperplane perpendicular to $l$ through p. See Fig. 2(b). $\mathscr{S}_{a}$ and $\mathscr{S}_{b}$ can touch both $S_{1}$ and $S_{2}$ only if $\mathrm{p} \in \operatorname{cl} S_{1} \cap \mathrm{cl} S_{2}$, which violates the assumption that $\mathrm{cl} S_{1} \cap \mathrm{cl} S_{2}=\varnothing$.

Case 3: $\mathscr{S}_{a}$ and $\mathscr{S}_{b}$ intersect in a nondegenerate lower-dimensional sphere. Let $\lambda$ be the hyperplane through the sphere of intersection. Note that $\lambda$ is parallel to $\pi_{w}$. If $\lambda \neq \pi_{\mathrm{w}}$, the sets of touching points of $\mathscr{S}_{a}$ and of $\mathscr{S}_{b}$ lie in opposite closed half-spaces bounded by $\lambda$. See Fig. 2(c). Therefore, since $\lambda$ is parallel to $\pi_{\mathrm{w}} \mathscr{S}_{a}$ cannot touch $S_{2}$ and/or $\mathscr{S}_{b}$ cannot touch $S_{1}$, which is a contradiction. Thus $\lambda=\pi_{w} . \mathscr{S}_{a}$ and $\mathscr{S}_{b}$ can each touch points in both $S_{1}$ and $S_{2}$ only if one of the touching points on $S_{1}$ and one of the touching points on $S_{2}$ are both on $\pi_{w}$. These touching points are contained in $\mathscr{S}_{a} \cap \mathscr{S}_{b}$. Let $\mathbf{q}$ be strictly between $\mathbf{q}_{a}$ and $\mathbf{q}_{b}$, and let $\mathscr{S}_{q}$ be the sphere centered at $\mathbf{q}$ and passing through $\mathscr{S}_{a} \cap \mathscr{S}_{b}$. See Fig. 2(d). Clearly the open ball $\mathscr{B}_{q}$ defined by $\mathscr{S}_{q}$ is contained in $\mathscr{A}_{a} \cup \mathscr{B}_{b}$ and hence is free of points of $S_{1} \cup S_{2}$. Thus $\mathscr{S}_{q}$ is a maximal sphere touching both $S_{1}$ and $S_{2}$ in $\pi_{\mathrm{w}}$, and $\mathrm{q} \in B\left(S_{1}, S_{2}\right)$.

The theorem follows directly.

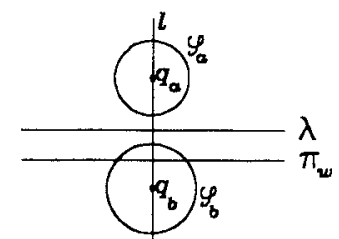

(a)

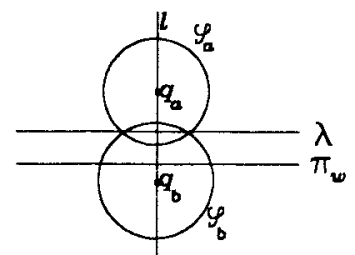

(c)
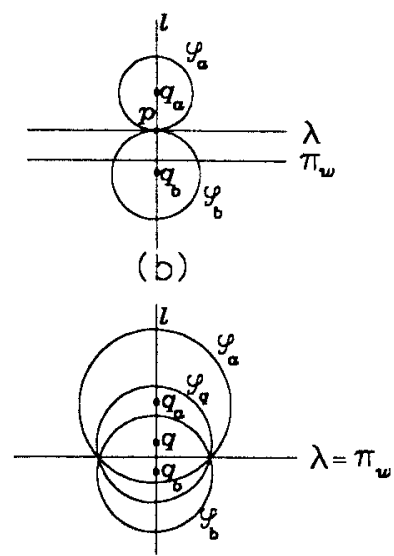

(d)

Fig. 2. Illustration of various cases for proof of Theorem 2. 


\subsection{Intersection with Every Perpendicular}

In Section 3.1 we proved that if the intersection between the bisector and a line perpendicular to a weak linear separator exists, then it is connected; in this section we give necessary and sufficient conditions for every line perpendicular to the weak linear separator to intersect the bisector.

Let $I=\left\{\mathrm{x} \in E^{d-1} ; \alpha_{i} \leq x_{i} \leq \beta_{i}, i=1,2, \ldots, d-1\right\}$, for real numbers $\alpha_{i}<$ $\beta_{i}, i=1,2, \ldots, d-1$, be a closed $(d-1)$-cell in $\pi_{\mathrm{w}}$.

Theorem 3. Every line perpendicular to $\pi_{\mathrm{w}}$ intersects $B\left(S_{1}, S_{2}\right)$ if and only if

(1) $S_{1}, S_{2} \not \subset \pi_{w}$, or

(2) $S_{2} \subset \pi_{\mathrm{w}}$ and $\mathrm{cl} S_{1} \cap \pi_{\mathrm{w}}=\varnothing$, or vice versa.

Moreover, if every line perpendicular to $\pi_{\mathrm{w}}$ intersects $B\left(S_{1}, S_{2}\right)$, then, for any $(d-1)$-cell $I$ in $\pi_{w}, B\left(S_{1}, S_{2}\right) \cap(I \times \mathbb{R})$ is bounded.

We prove Theorem 3 by showing the sufficient conditions in Lemmas 1 and 2 and the necessary condition in Lemma 3.

Lemma 1. If $S_{1}, S_{2} \not \subset \pi_{w}$, then every line perpendicular to $\pi_{w}$ intersects $B\left(S_{1}, S_{2}\right)$. Moreover, $B\left(S_{1}, S_{2}\right) \cap(I \times \mathbb{R})$ is bounded.

Proof. Let $\mathbf{q}$ be a point on the line $l=\left\{\mathbf{x}: x_{i}=q_{i}, i=1, \ldots, d-1\right\}$ perpendicular to $\pi_{\mathbf{w}}$. Define $f(\mathbf{q})=d^{2}\left(\mathbf{q}, S_{1}\right)-d^{2}\left(\mathbf{q}, S_{2}\right)$, and observe that $\mathbf{q} \in B\left(S_{1}, S_{2}\right)$ if and only if $f(\mathbf{q})=0$. First assume that $q_{d}>0$. Let $\mathbf{p} \in S_{1}, p_{d}>0$; such a point must exist since $S_{1} \not \subset \pi_{w}$. See Fig. 3. Since $d^{2}\left(\mathbf{q}, S_{1}\right) \leq d^{2}(\mathbf{q}, \mathbf{p})=\sum_{i=1}^{d}\left(p_{i}-q_{i}\right)^{2}$ and $d^{2}\left(\mathbf{q}, S_{2}\right) \geq d^{2}\left(\mathbf{q}, \pi_{w}\right)=q_{d}^{2}, \quad f(\mathbf{q}) \leq \sum_{i=1}^{d}\left(p_{i}-q_{i}\right)^{2}-q_{d}^{2}=\sum_{i=1}^{d-1}\left(p_{i}-q_{i}\right)^{2}+p_{d}^{2}-$ $2 p_{d} q_{d}$. For sufficiently large $q_{d}, f(q)<0$. By a symmetric argument, for sufficiently small $q_{d}<0, f(\mathbf{q})>0$. But $d\left(\mathbf{p}, S_{i}\right)$ is a continuous function of $\mathbf{p}$ (Theorem 3 ,

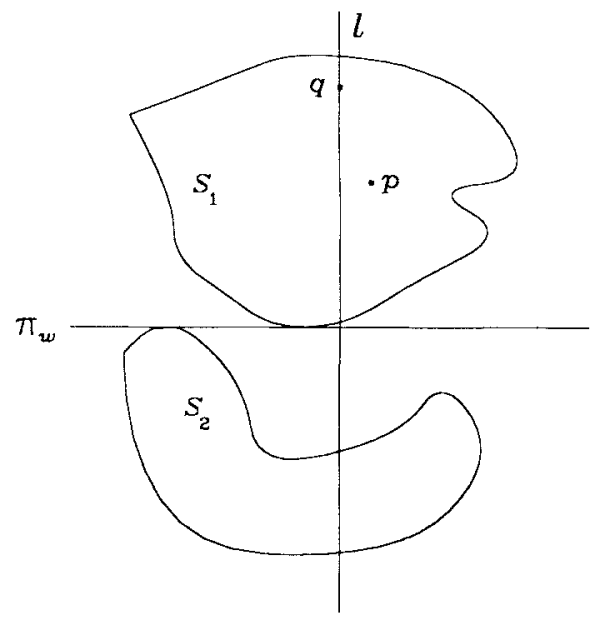

Fig. 3. Selecting points $p$ and $\mathbf{q}$ for the proof of Lemma 1. 
Section 1.8, of [4]). Therefore, since $f$ changes sign, it must have at least one zero, which implies that the line $l$ intersects $B\left(S_{1}, S_{2}\right)$.

It remains to show that $B\left(S_{1}, S_{2}\right) \cap(I \times \mathbb{R})$ is bounded. Observe that for $q_{d}>\left(\sum_{i=1}^{d-1}\left(p_{i}-q_{i}\right)^{2}+p_{d}^{2}\right) / 2 p_{d}, f(\mathbf{q})<0$. Thus the intersection of $B\left(S_{1}, S_{2}\right)$ with $l$ is bounded in the positive $x_{d}$-direction by a continuous function of $q_{1}, \ldots, q_{d-1}$. It is similarly bounded in the negative $x_{d}$-direction. In particular, as $I$ is compact, $B\left(S_{1}, S_{2}\right) \cap(I \times \mathbb{R})$ is bounded.

Lemma 2. If $S_{2} \subset \pi_{\mathrm{w}}$ and $\mathrm{cl} S_{1} \cap \pi_{\mathrm{w}}=\varnothing$, then every line perpendicular to $\pi_{\mathrm{w}}$ intersects $B\left(S_{1}, S_{2}\right)$. Moreover, $B\left(S_{1}, S_{2}\right) \cap(I \times \mathbb{R})$ is bounded.

Proof. Again, let $\mathbf{q}$ be a point on a line $l$ perpendicular to $\pi_{w}$ and vary $q_{d}$ so that $\mathbf{q}$ moves along $l$. By the arguments in the proof of Lemma $1, f(\mathbf{q})=d^{2}\left(\mathbf{q}, S_{1}\right)-$ $d^{2}\left(\mathbf{q}, S_{2}\right)<0$ for sufficiently large $q_{d}>0$.

We now show that there exists a $q_{d} \leq 0$ such that $f(\mathbf{q}) \geq 0$. Let $\mathbf{u}=\ln \pi_{\mathbf{w}}$ and $\mathbf{p}$ be a point of $\mathrm{cl} S_{2}$ closest to $\mathbf{u}$. If $\mathbf{p}=\mathbf{u}$, then $d^{2}\left(\mathbf{q}, S_{2}\right)=q_{d}^{2}$. Therefore, since $d^{2}\left(\mathbf{q}, S_{1}\right)>d^{2}\left(\mathbf{q}, \pi_{\mathrm{w}}\right)=q_{d}^{2}$ for all $q_{d} \leq 0, f(\mathbf{q})>0$ for all $q_{d} \leq 0$.

If $\mathbf{p} \neq \mathbf{u}$, then consider an open ball $\mathbf{B}_{\mathbf{u}}$ of radius $d(\mathbf{u}, \mathbf{p})$ centered at $\mathbf{u}$. If cl $S_{1} \cap \mathrm{cl} \mathbf{B}_{\mathbf{v}}=\varnothing$, then $d^{2}\left(\mathbf{u}, S_{1}\right)>d^{2}\left(\mathbf{u}, S_{2}\right)$, which leads to $f(\mathbf{u})>0$. Otherwise, since $\mathrm{cl} S_{1} \cap \mathrm{cl} \mathbf{B}_{\mathbf{u}}$ is compact, there exists a point $\mathbf{t} \in \mathrm{cl} S_{1} \cap \mathrm{cl} \mathbf{B}_{\mathbf{u}}$ with smallest $x_{d}$-coordinate. Moreover, $t_{d}>0$ because cl $S_{1} \cap \pi_{w}=\varnothing$. See Fig. 4. For $q_{d} \leq 0$, $d^{2}\left(\mathbf{q}, S_{1}\right) \geq\left(t_{d}-q_{d}\right)^{2}$. Therefore, since $d^{2}\left(\mathbf{q}, S_{2}\right)=\sum_{i=1}^{d}\left(p_{i}-q_{i}\right)^{2}, f(\mathbf{q}) \geq t_{d}^{2}-$ $2 t_{d} q_{d}-\sum_{i=1}^{d-1}\left(p_{i}-q_{i}\right)^{2}$ if $q_{d} \leq 0$. For sufficiently small $q_{d} \leq 0, f(\mathbf{q}) \geq 0$. Thus $f(\mathbf{q})$ has a zero and the intersection result follows. Boundedness is guaranteed, since $t$ is confined to a bounded set as long as $\mathbf{u} \in I$.

To complete the proof of Theorem 3, it remains to prove the necessary condition.

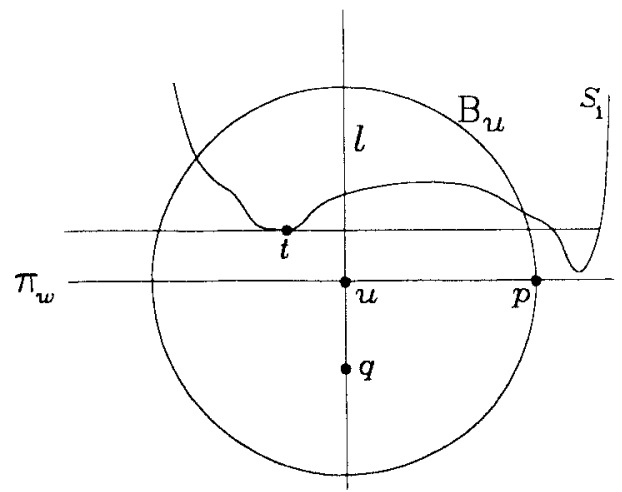

Fig. 4. Construction for proof of Lemma 2. 


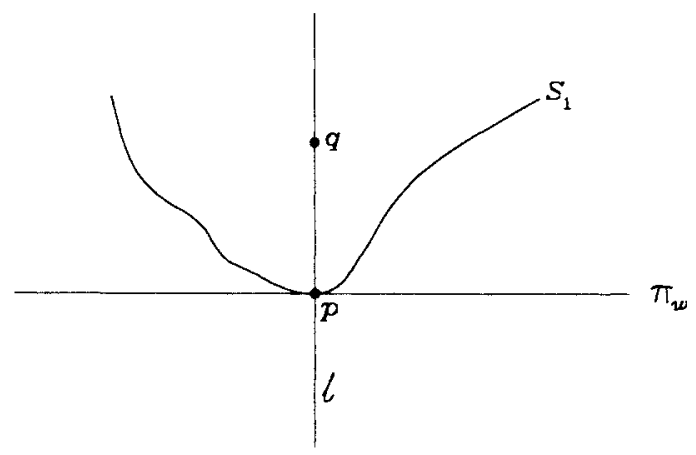

Fig. 5. Construction for proof of Lemma 3.

Lemma 3. If $S_{2} \subset \pi_{\mathrm{w}}$ and $\mathrm{cl} S_{1} \cap \pi_{\mathrm{w}} \neq \varnothing$, then there exist lines perpendicular to $\pi_{\mathrm{w}}$ that do not intersect $B\left(S_{1}, S_{2}\right)$.

Proof. Consider the line $l$ perpendicular to $\pi_{\mathrm{w}}$ through a point $\mathrm{p} \in \mathrm{cl} S_{1} \cap \pi_{\mathrm{w}}$. See Fig. 5. For any $\mathbf{q} \in l, d^{2}\left(\mathbf{q}, S_{1}\right) \leq d^{2}(\mathbf{q}, \mathbf{p})=q_{d}^{2}$. Since $\mathbf{p} \notin \operatorname{cl} S_{2}$ and $S_{2} \subset \pi_{\mathbf{w}}$, $d^{2}\left(\mathbf{q}, S_{2}\right)>q_{d}^{2}$. Therefore $d^{2}\left(\mathbf{q}, S_{1}\right)<d^{2}\left(\mathbf{q}, S_{2}\right), \forall \mathbf{q} \in l$, so $l$ cannot intersect $B\left(S_{1}, S_{2}\right)$.

Figure 6 shows two examples as an illustration of Lemma 3. From Theorems 2 and 3 we have:

Corollary 1. If $S_{1}$ and $S_{2}$ are weakly linearly separated by $\pi_{\mathrm{w}}$ and $\mathrm{cl} S_{1} \subset \pi_{\mathrm{w}}^{1}$, then every line perpendicular to $\pi_{\mathrm{w}}$ intersects $B\left(S_{1}, S_{2}\right)$ in a single point.

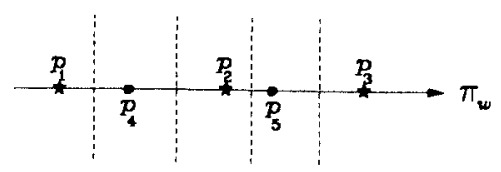

(a)

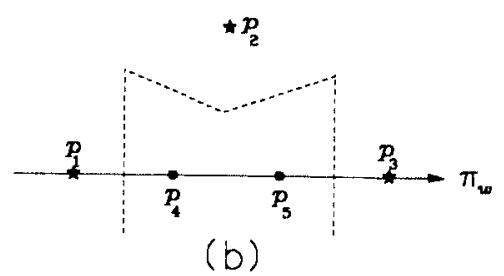

Fig. 6. Sets illustrating the condition of Lemma 3. Let $S_{1}=\left\{\mathbf{p}_{1}, \mathbf{p}_{2}, \mathbf{p}_{3}\right\}$ and $S_{2}=\left\{\mathbf{p}_{4}, \mathbf{p}_{5}\right\}$. (a) Both $S_{1}$ and $S_{2}$ are completely contained in their weak linear separator $\pi_{w} . B\left(S_{1}, S_{2}\right)$ consists of just four lines (shown dashed), all of which are perpendicular to $\pi_{w}$. (b) $S_{1}$ is only partially contained in $\pi_{w}$. In both cases, there exist lines perpendicular to $\pi_{\mathrm{w}}$ that do not intersect $B\left(S_{1}, S_{2}\right)$. 
As a special case we also have:

Corollary 2. If a hyperplane $\pi$ is a linear separator of $S_{1}$ and $S_{2}$, then every line perpendicular to $\pi$ intersects $B\left(S_{1}, S_{2}\right)$ in a single point.

\subsection{Intersection with a Specific Perpendicular}

Theorem 3 gave the necessary and sufficient conditions under which every line perpendicular to $\pi_{w}$ intersects $B\left(S_{1}, S_{2}\right)$. Even if such global conditions do not hold, it is still possible to obtain local results. This section gives necessary and sufficient conditions under which any specific line perpendicular to $\pi_{w}$ intersects $B\left(S_{1}, S_{2}\right)$.

Lemma 4. Let $S_{1}, S_{2} \subset \pi_{\mathrm{w}}$ and let $\mathbf{u}$ be a point in $\pi_{\mathrm{w}}$. A line l perpendicular to $\pi_{\mathrm{w}}$ and passing through $\mathbf{u}$ intersects $B\left(S_{1}, S_{2}\right)$ if and only if $d\left(\mathbf{u}, S_{1}\right)=d\left(\mathbf{u}, S_{2}\right)$. Moreover, if l intersects $B\left(S_{1}, S_{2}\right)$, then $l \subset B\left(S_{1}, S_{2}\right)$.

Proof. Let $\mathbf{q} \in l$. Then $d^{2}\left(\mathbf{q}, S_{i}\right)=d^{2}\left(\mathbf{u}, S_{i}\right)+q_{d}^{2}$, for $i=1,2$. Therefore, if $d\left(\mathbf{u}, S_{1}\right)=d\left(\mathbf{u}, S_{2}\right)$, then $d\left(\mathbf{q}, S_{1}\right)=d\left(\mathbf{q}, S_{2}\right)$, which implies that $l \subset B\left(S_{1}, S_{2}\right)$. Conversely, if $\mathbf{q} \in B\left(S_{1}, S_{2}\right)$, then $d^{2}\left(\mathbf{q}, S_{1}\right)=d^{2}\left(\mathbf{q}, S_{2}\right)$ and hence $d\left(\mathbf{u}, S_{1}\right)=$ $d\left(\mathbf{u}, S_{2}\right)$.

Lemma 5. Let $S_{2} \subset \pi_{\mathrm{w}}, S_{1} \not \subset \pi_{\mathrm{w}}$, cl $S_{1} \cap \pi_{\mathrm{w}} \neq \varnothing$, and $\mathbf{u} \in \pi_{\mathrm{w}}$ such that $d\left(\mathbf{u}, S_{2}\right) \neq$ $d\left(\mathbf{u}, \mathrm{cl} S_{1} \cap \pi_{\mathrm{w}}\right)$. A line $l$ perpendicular to $\pi_{\mathrm{w}}$ and passing through $\mathbf{u}$ intersects $B\left(S_{1}, S_{2}\right)$ if and only if $d\left(\mathbf{u}, S_{2}\right)<d\left(\mathbf{u}, \mathrm{cl} S_{1} \cap \pi_{\mathbf{w}}\right)$. Moreover, if $l$ intersects $B\left(S_{1}, S_{2}\right)$, then it does so in a single point.

Proof. Let $\mathbf{q}$ be a point in $l$. See Fig. 7. Sufficiency follows from arguments similar to those of the proof of Lemma 2. To show necessity, assume that $q \in B\left(S_{1}, S_{2}\right)$. Since $\quad d^{2}\left(\mathbf{q}, S_{1}\right)=d^{2}\left(\mathbf{q}, S_{2}\right), \quad d^{2}\left(\mathbf{q}, S_{2}\right)=d^{2}\left(\mathbf{u}, S_{2}\right)+q_{d}^{2}, \quad d^{2}\left(\mathbf{q}, \mathrm{cl} S_{1} \cap \pi_{\mathrm{w}}\right)=$ $d^{2}\left(\mathbf{u}, \mathrm{cl} S_{1} \cap \pi_{\mathrm{w}}\right)+q_{d}^{2}$, and $d^{2}\left(\mathbf{q}, S_{1}\right) \leq d^{2}\left(\mathbf{q}, \mathrm{cl} S_{1} \cap \pi_{\mathrm{w}}\right)$, we have $d^{2}\left(\mathbf{u}, S_{2}\right)+q_{d}^{2} \leq$ $d^{2}\left(\mathbf{u}, \mathrm{cl} S_{1} \cap \pi_{\mathrm{w}}\right)+q_{d}^{2}$. Necessity follows since, by hypothesis, $d\left(\mathbf{u}, S_{2}\right) \neq$ $d\left(\mathbf{u}, \mathrm{cl} S_{1} \cap \pi_{\mathrm{w}}\right)$.

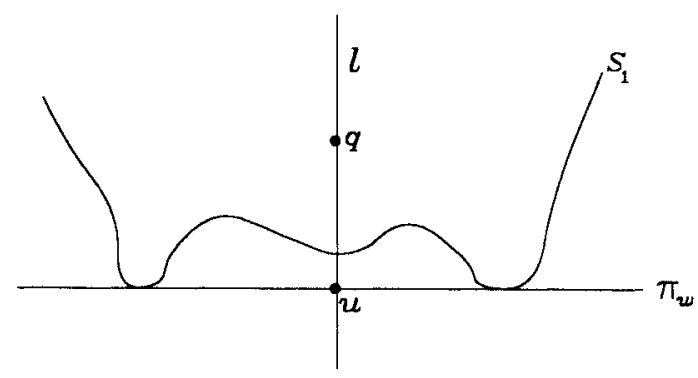

Fig. 7. Construction for proof of Lemma 5. 
To see that the intersection is a single point, assume the contrary. Then, by Theorem 2, there exists $q \in \operatorname{int}\left[l \cap B\left(S_{1}, S_{2}\right)\right]$ such that the touching points of the maximal sphere centered at $\mathbf{q}$ are contained in $\pi_{\mathbf{w}}$. This implies that the touching points in $S_{1}$ are in $\mathrm{cl} S_{1} \cap \pi_{\mathrm{w}}$ and that $d^{2}\left(\mathbf{q}, S_{2}\right)=d^{2}\left(\mathbf{q}, \mathrm{cl} S_{1} \cap \pi_{\mathrm{w}}\right)-$ a contradiction.

Let $\mathbf{B}$ be an open $(d-1)$-dimensional ball and let $\mathbf{v}$ be a point on a line perpendicular to the hyperplane that contains $\mathbf{B}$, and passing through the center of B. The truncated semicone $C(\mathbf{v}, \mathbf{B})$ is defined to be int $\mathrm{CH}(\mathbf{v} \cup \mathbf{B})$.

Lemma 6. Let $S_{2} \subset \pi_{\mathrm{w}}, S_{1} \not \subset \pi_{\mathrm{w}}$, cl $S_{1} \cap \pi_{\mathrm{w}} \neq \varnothing$, and $\mathbf{u} \in \pi_{\mathrm{w}}$ such that $d\left(\mathbf{u}, S_{2}\right)=$ $d\left(\mathbf{u}, \mathrm{cl} S_{1} \cap \pi_{\mathbf{w}}\right)$. Let $\mathbf{B} \subset \pi_{\mathbf{w}}$ be the $(d-1)$-dimensional ball of radius $d\left(\mathbf{u}, S_{2}\right)$ centered at $\mathbf{u}$. $A$ line l perpendicular to $\pi_{w}$ and passing through $\mathbf{u}$ intersects $B\left(S_{1}, S_{2}\right)$ if and only if there exists a point $\mathbf{q} \in \ln \pi_{w}^{1}$ such that $C(\mathbf{q}, \mathbf{B}) \cap S_{1}=\varnothing$. Furthermore, if l intersects $B\left(S_{1}, S_{2}\right)$, then a half-line of $l$ is contained in $B\left(S_{1}, S_{2}\right)$.

Proof. To show sufficiency, let there be a point $\mathbf{q}_{1} \in l \cap \pi_{\mathrm{w}}^{1}$ such that $C\left(\mathbf{q}_{1}, \mathbf{B}\right) \cap S_{1}=\varnothing$. See Fig. 8. Now, consider the one-parameter family of $d$-dimensional balls (and the associated boundary spheres) the intersect $\pi_{w}$ in $\mathbf{B}$ and whose centers lie on $l$. Some of these boundary spheres must intersect $\pi_{\mathrm{w}}^{1}$ within $C\left(\mathbf{q}_{1}, \mathbf{B}\right)$. Since B intersects neither $\mathrm{cl} S_{1}$ nor $\mathrm{cl} S_{2}$, but the boundary of B touches both $\mathrm{cl} S_{1}$ and $\mathrm{cl} S_{2}$, such spheres must be maximal spheres. Furthermore, any member of the family whose center has smaller $x_{d}$-coordinate must also be maximal. Thus, a half-line of $l$ is contained in $B\left(S_{1}, S_{2}\right)$.

To show necessity, consider a maximal sphere $\mathscr{S}$ (and the associated ball $\mathscr{B}$ ) centered at $\mathbf{q} \in l \cap B\left(S_{1}, S_{2}\right)$. Note that $\mathbf{u} \notin \operatorname{cl}\left(S_{1} \cup S_{2}\right)$ because $d\left(\mathbf{u}, S_{2}\right)=$ $d\left(\mathbf{u}, \mathrm{cl} S_{1} \cap \pi_{\mathrm{w}}\right)$ and $\mathrm{cl} S_{1} \cap \mathrm{cl} S_{2}=\varnothing$. Therefore, $\exists \mathbf{q}_{2} \in \pi_{\mathrm{w}}^{1} \cap l \cap \mathscr{S}$. See Fig. 8 . Since $C\left(\mathbf{q}_{2}, \mathbf{B}\right) \subset \mathscr{B}, C\left(\mathbf{q}_{2}, \mathbf{B}\right) \cap S_{1}=\varnothing$.

An example where the conditions of Lemma 6 do not hold is shown in Fig. 9.

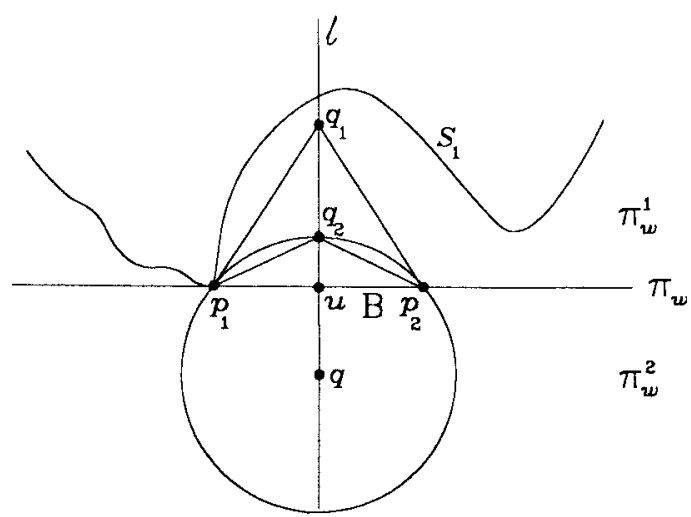

Fig. 8. Construction for proof of Lemma 6. 


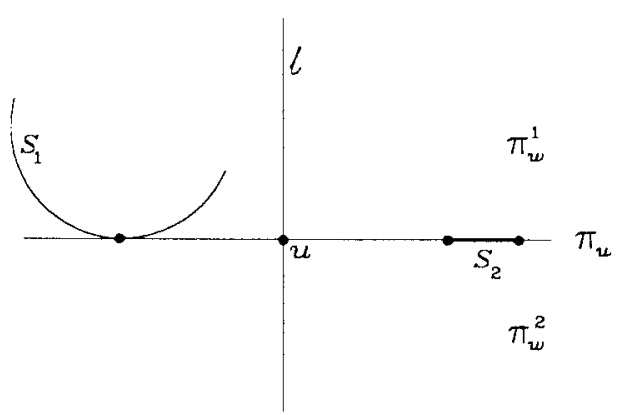

Fig. 9. An example where the conditions of Lemma 6 do not hold. $S_{1}$ is an arc of a circle, $S_{2} \subset \pi_{w}$ and $d\left(\mathbf{u}, S_{2}\right)=d\left(\mathbf{u}, \mathrm{cl} S_{1} \cap \pi_{\mathrm{w}}\right)$. The line $l$ will not intersect $B\left(S_{1}, S_{2}\right)$.

Necessary and sufficient conditions for any specific line perpendicular to $\pi_{\mathrm{w}}$ to intersect $B\left(S_{1}, S_{2}\right)$ follow directly from Theorem 3 and Lemmas 4-6.

Theorem 4. Let $\mathbf{u} \in \pi_{\mathrm{w}}$ and let l be the line perpendicular to $\pi_{\mathrm{w}}$ passing through $\mathbf{u}$. The line lintersects $B\left(S_{1}, S_{2}\right)$ if and only if (up to a switch of $S_{1}$ and $S_{2}$ )

1. $S_{1} \not \subset \pi_{\mathrm{w}}$ and $S_{2} \not \subset \pi_{\mathrm{w}}$, or

2. $S_{2} \subset \pi_{\mathrm{w}}$, and $\mathrm{cl} S_{1} \cap \pi_{\mathrm{w}}=\varnothing$, or

3. $S_{1} \subset \pi_{\mathrm{w}}, S_{2} \subset \pi_{\mathrm{w}}$ and $d\left(\mathbf{u}, S_{1}\right)=d\left(\mathbf{u}, S_{2}\right)$, or

4. $S_{2} \subset \pi_{\mathrm{w}}, S_{1} \not \subset \pi_{\mathrm{w}}, \mathrm{cl} S_{1} \cap \pi_{\mathrm{w}} \neq \varnothing$, and $d\left(\mathbf{u}, S_{2}\right)<d\left(\mathbf{u}, \mathrm{cl} S_{1} \cap \pi_{\mathrm{w}}\right)$, or

5. $S_{2} \subset \pi_{\mathrm{w}}, S_{1} \not \subset \pi_{\mathrm{w}}, \mathrm{cl} S_{1} \cap \pi_{\mathrm{w}} \neq \varnothing, d\left(\mathrm{u}, S_{2}\right)=d\left(\mathrm{u}, \mathrm{cl} S_{1} \cap \pi_{\mathrm{w}}\right)$, and there exists a point $\mathbf{q} \in l \cap \pi_{w}^{1}$ such that $C(\mathbf{q}, \mathbf{B}) \cap S_{1}=\varnothing$, where $\mathbf{B} \subset \pi_{\mathrm{w}}$ is the open $(d-1)$-dimension ball of radius $d\left(\mathbf{u}, S_{2}\right)$ centered at $\mathbf{u}$.

\section{Continuous Mapping from Linear Separator to Bisector}

We have thus far shown exactly when a line perpendicular to $\pi_{\mathrm{w}}$ intersects $B\left(S_{1}, S_{2}\right)$ at a single point. This defines a mapping which lifts points of the separator up to the bisector. We now show that wherever such a mapping exists, it is continuous. Notice that this map will automatically be a homeomorphism, as its inverse is the orthogonal projection onto the separator - certainly a well-defined continuous map.

Theorem 5. Let the hyperplane $\pi_{\mathrm{w}}: x_{d}=0$ be a weak linear separator of $S_{1}$ and $S_{2}$ and let $M$ be a relatively open subset of $\pi_{\mathrm{w}}$ such that $\forall \mathbf{p} \in M$, the line through $\mathbf{p}$ perpendicular to $\pi_{\mathrm{w}}$ intersects $B\left(S_{1}, S_{2}\right)$ in a single point. Then the mapping $b: M \rightarrow \mathbb{B}$ such that $\left(x_{1}, \ldots, x_{d-1}, b\left(x_{1}, \ldots, x_{d-1}\right)\right) \in B\left(S_{1}, S_{2}\right)$ is continuous.

To prove this we use the following lemma. Let $E$ and $F$ be topological spaces. A function $g: E \rightarrow F$ is said to have a closed graph if its graph $\{(x, y): y=g(x), x \in E\}$ in the product space $E \times F$ is a closed set. 
Lemma 7 (Theorem 3, Chapter VII of [3]). Let $E, F$ be topological spaces and let $g: E \rightarrow F$ be a function. If $g$ has a closed graph and $F$ is compact, then $g$ is continuous.

Proof of Theorem 5. The mapping $b$ is a function by hypothesis. We show that $b$ is continuous at an arbitrary $\mathbf{p} \in M$. Let $I=\left\{\mathbf{x} \in E^{d-1} ; \alpha_{i} \leq x_{i} \leq \beta_{i}, i=1,2, \ldots, d-\right.$ $1\}$, for real numbers $\alpha_{i}<\beta_{i}, i=1,2, \ldots, d-1$, be a closed $(d-1)$-cell in $M$ containing $\mathbf{p}$ in its interior and let $b_{\mathrm{I}}$ denote the restriction of $b$ to $I$. We claim that $b_{1}$ has a compact graph and thus, by Lemma $7, b$ is continuous at $\mathbf{p}$.

The graph of $b_{1}$ is the intersection of $B\left(S_{1}, S_{2}\right)$ and $I \times \mathbb{R}$. Referring to the proof of Lemma $1, B\left(S_{1}, S_{2}\right)=f^{-1}(0)$. Since the inverse image of a closed set under a continuous map is closed (p. 35 of [4]), $B\left(S_{1}, S_{2}\right)$ is closed. Therefore, the graph of $b_{I}$ is closed.

It remains to show that the range of $b$ is also bounded and, hence, compact. Since the line perpendicular to each point in $M$ intersects $B\left(S_{1}, S_{2}\right)$, one of the five conditions of Theorem 4 must hold for each point in $M$. Furthermore, since by hypothesis, each such line intersects $B\left(S_{1}, S_{2}\right)$ in a single point, Lemmas 4 and 6 imply that, up to a switch of $S_{1}$ and $S_{2}$, one of the following must hold:

1. $S_{1} \not \subset \pi_{\mathrm{w}}$ and $S_{2} \not \subset \pi_{\mathrm{w}}$, or

2. $S_{2} \subset \pi_{\mathrm{w}}$, and cl $S_{1} \cap \pi_{\mathrm{w}}=\varnothing$, or

3. $S_{2} \subset \pi_{\mathrm{w}}, S_{1} \not \subset \pi_{\mathrm{w}}, \mathrm{cl} S_{1} \cap \pi_{\mathrm{w}} \neq \varnothing$, and $d\left(\mathbf{u}, S_{2}\right)<d\left(\mathbf{u}, \operatorname{cl} S_{1} \cap \pi_{\mathrm{w}}\right), \forall \mathbf{u} \in M$.

In the first two cases, Theorem 3 establishes that the graph is bounded. In the third case, Lemma 5 and the fact that $I$ is compact establish that the graph is bounded. Therefore, the graph of $b_{I}$ is compact, which implies that $b$ is continuous at every point of $M$.

When $\pi_{\mathrm{w}}$ is a linear separator, we have:

Corollary 3. Let the hyperplane $\pi: x_{d}=0$ be a linear separator of $S_{1}$ and $S_{2}$. If $b$ is the mapping $b: \pi \rightarrow \mathbb{R}$ such that $\left(x_{1}, \ldots, x_{d-1}, b\left(x_{1}, \ldots, x_{d-1}\right)\right) \in B\left(S_{1}, S_{2}\right)$, then $b$ is a continuous function. In fact, the perpendicular projection of $B\left(S_{1}, S_{2}\right)$ onto $\pi$ is $a$ homeomorphism.

Corollary 3 generalizes the notion described in [8] that the bisector of linearly separated point sites in $E^{2}$ is a monotone chain. More importantly, it shows that $B\left(S_{1}, S_{2}\right)$ is a $(d-1)$-manifold in $E^{d}$.

\section{Summary}

In this paper we have presented some general properties of bisectors of sets in $E^{d}$ that are separated by hyperplanes. We have given necessary and sufficient conditions for the perpendicular projection of the bisector of two weakly linearly separated sets onto a separator to be a homeomorphism. This study needs to be expanded in two major directions.

Throughout this paper we have required that $\operatorname{cl} S_{1} \cap \operatorname{cl} S_{2}=\varnothing$. When the closures of the sets are not disjoint, the bisector need not be a manifold, as 


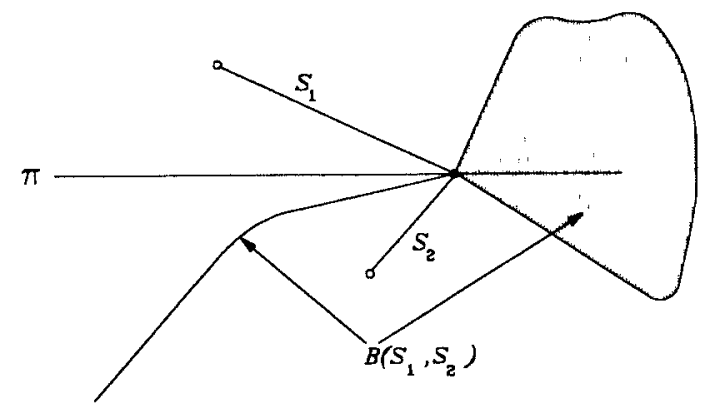

Fig. 10. A nonhomogeneously two-dimensional bisector. $B\left(S_{1}, S_{2}\right)$ is the bisector between two open line-segments that do not intersect. However, the closures of the line-segments intersect at an endpoint.

illustrated in Fig. 10. This issue has been addressed in the literature on Voronoi diagrams of point sites and open line segments in $E^{2}$ by defining the bisectors between individual elements $a b$ initio so that they are always homogeneously onedimensional [5]-[7], [9]. We think that this needs further investigation.

Another direction in which the theory could be generalized is to investigate the general conditions under which the bisector of two sets is a $(d-1)$-manifold that partitions $E^{d}$ into two disjoint regions. In this paper we have shown that if the two sets are linearly separable, then their bisector has this property. Also, some sufficient conditions for the bisector to be a simple closed curve were given in [9] for sets in $E^{2}$ that are not even weakly linearly separable. We are not aware of any other result related to this problem.

\section{Acknowledgments}

We thank Michael O'Connor for helping us to formalize the proof of Lemma 1 and for suggesting the current proof of Theorem 5, which is much simpler than our original proof. We also thank him for encouraging us to generalize our results in $E^{2}$

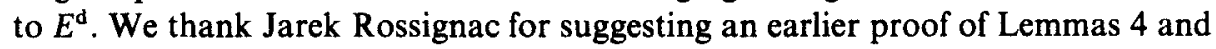
5 , and the referee for suggesting several important improvements.

\section{References}

1. F. L. Bookstein, The Line-Skeleton, Comput. Graphics and Image Process., vol. 11, pp. 123-137, October 1979.

2. L. Guibas and J. Stolfi, Primitives for the Manipulation of General Subdivisions and the Computation of Voronoi Diagrams, ACM Trans. Graphics, vol. 4, no. 2, pp. 74-123, April 1985.

3. T. Husain, Topology and Maps, New York: Plenum, 1977.

4. P. Kelly and M. Weiss, Geometry and Convexity: A Study in Mathematical Methods, New York: Wiley, 1979.

5. D. T. Lee, Medial Axis Transformation of a Planar Shape, IEEE Trans. Pattern Anal. Mach. Intell, vol. 4, no. 4, pp. 363-369, July 1982. 
6. D. T. Lee and R. L. Drysdale, III, Generalization of Voronoi Diagrams in the Plane, SIAM $J$. Comput., vol. 10, no. 1, pp. 73-87, February 1981.

7. D. Leven and M. Sharir, Intersection and Proximity Problems and Voronoi Diagrams, in J. T. Schwartz and C. Yap, editors, Algorithmic and Geometric Aspects of Robotics, pp. 187-228, Hillsdale, New Jersey: Erlbaum, 1987.

8. F. P. Preparata and M. I. Shamos, Computational Geometry: An Introduction, New York: SpringerVerlag, 1985.

9. V. Srinivasan and L. R. Nackman, Voronoi Diagram for Multiply-Connected Polygonal Domains I: Algorithm, IBM J. Res. Develop., vol. 31, no. 3, pp. 361-372, May 1987.

Received November 3, 1988, and in revised form March 22, 1989, and January 24, 1990. 